\title{
ROLE OF INERTIAL EFFECTS IN ELECTRON TRANSFER KINETICS
}

\author{
P. GAJDEK
}

Faculty of Chemistry, Jagiellonian University, 3 Ingardena, 30-060 Kraków, Poland

(Received February 13, 1996; revised version April 23, 1996)

The electron transfer rates at the steady state are evaluated in terms of the Gaussian wave packet motion on free energy curves in the two- and three-surface models in the presence of inertial effects. The autocorrelation functions of the solvent polarization coordinate are fitted to the results of recent molecular dynamics simulations. It is found that the inertial effects are particularly important for the electron transfer processes in acetonitrile and water. They constitute an impeding factor in the wave packet motion. The neglect of the inertial part of the solvent autocorrelation function gives underestimation of the electron transfer rate coefficient.

PACS numbers: 82.20.-w, 82.20.Pm

\section{Introduction}

Over the past decade there have been many theoretical and experimental investigations concerning the role of solvent dynamics in electron transfer [1] in a picosecond and subpicosecond time domain. The femtosecond time scale solvent dynamics, however, has not been accessible by experiment up to recent years. The time dependent fluorescence Stokes shift (TDFSS) experiments determined the solvent autocorrelation function $\Delta(t)$

$$
\Delta(t)=\frac{\nu(t)-\nu(\infty)}{\nu(0)-\nu(\infty)}
$$

where $\nu(t), \nu(\infty), \nu(0)$ are the fluorescence frequencies at times $t, \infty$ and 0 . In the picosecond time scale this function was analytically represented as either a single exponential or a sum of exponentials with characteristic solvation times $\tau_{i}(i=1,2 \ldots)[2]$

$$
\Delta_{1}(t)=\sum_{i} a_{i} \exp \left(-t / \tau_{i}\right), \quad \sum_{i} a_{i}=1
$$

Rosenthal et al. [3] first observed a very fast contribution to the solvent response. Such form of solvation dynamics was theoretically predicted [4-6]. In the light of 
recent investigations the ultrafast Gaussian inertial component of $\Delta(t)$ should be also taken into account [7]

$$
\Delta_{2}(t)=\sum_{i} a_{i}^{\prime} \exp \left(-t / \tau_{i}\right)+b \exp \left(-\omega^{2} t^{2} / 2\right), \quad \sum_{i} a_{i}^{\prime}+b=1,
$$

where $\omega$ is the characteristic vibrational frequency of a given solvent.

During the last few years the very large evidence on solvation dynamics in polar solvents has been acquired [8] by means of the molecular dynamics (MD) computer experiments in water [9-14], solvent resembling methyl chloride [15, 16], acetonitrile [17] and methanol [18-20]. In acetonitrile (no hydrogen bonding), the initial Gaussian decay of $\Delta(t)$ constitutes $70-80 \%$ of the total relaxation and occurs on a time scale of $100-250 \mathrm{fs}$. In methanol and water, the inertial decay occurs on a much shorter time scale (ca. $30 \mathrm{fs}$ ) than in aprotic solvents. Librations, resulting from the hydrogen bond restoring forces, come into play at an early stage of the relaxation and break this initial inertial decay [20]. In water we observe that the Gaussian decay of $\Delta(t)$ constitutes $50-80 \%$ of the total response, while in methanol it accounts for only $10-20 \%$. The initial decay of $\Delta(t)$ for methanol is determined by a hydrogen bond rotation. The key difference is that in water there are three distinct ways of performing this rotational motion (with nearly equal moments of inertia), while in methanol there is only a single way [20]. All the three situations with increasing polarity: in methanol (a), acetonitrile (b) and water (c) are presented in Fig. 1.

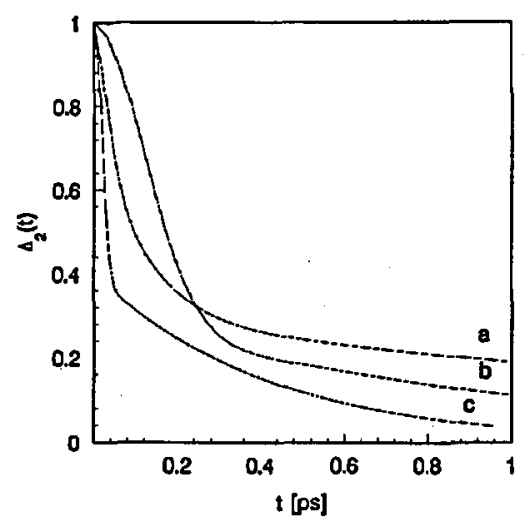

Fig. 1. The autocorrelation functions of the solvent polarization coordinate $\Delta_{2}(t)$ in the cases of (a) methanol, (b) acetonitrile, (c) water.

The outline of this paper is as follows. First, main theoretical concepts concerning the two- [21-23] and three-surface [24-26] models of an electron transfer process in solution are given, subsequently the dependences of the time-scale functions [26-28] and the electron transfer rates in the two-surface model, as well as in the three-surface model, on the free energy for methanol, acetonitrile and water including the inertial effects are analyzed. 


\section{Two- and three-surface models}

The dynamics in the reaction coordinate $q$ is described in terms of the Gaussian wave packet $\phi\left(q, q^{\prime}, t\right)$ for a harmonic system in an overdamped limit [28]: This function represents the solution of the following equation:

$$
\left(\partial / \partial t-L_{\text {eff }}\right) \phi\left(q, q^{\prime}, t\right)=0
$$

with initial condition $\phi\left(q, q^{\prime}, 0\right)=\delta\left(q-q^{\prime}\right) . L_{\text {eff }}$ is the effective time-independent diffusion operator. The solution of Eq. (4), $\phi\left(q, q^{\prime}, t\right)$, is given in terms of the time correlation function of the reaction coordinate, $\Delta(t)=\langle\delta q(0) \delta q(t)\rangle /\left\langle\delta q(0)^{2}\right\rangle$, by the following equation:

$$
\phi\left(q, q^{\prime}, t\right)=\left\{2 \pi\left\langle q^{2}\right\rangle\left[1-\Delta(t)^{2}\right]\right\}^{-1 / 2} \exp \left\{-\frac{\left[q-q^{\prime} \Delta(t)\right]^{2}}{2\left\langle q^{2}\right\rangle\left[1-\Delta(t)^{2}\right]}\right\},
$$

where $\left\langle q^{2}\right\rangle=2 \lambda k_{\mathrm{B}} T$. The function $\phi\left(q, q^{\prime}, t\right)$ gives the conditional probability that the reaction coordinate would assume the value $q$ at a time $t$, provided it had the value $q^{\prime}$ at $t=0$ and the system is on the single potential surface. For a very long-time limit $\phi\left(q, q^{\prime}, t\right)$ approaches the equilibrium distribution $\phi_{\mathrm{eq}}(q)$. It is convenient to write the Laplace transform $\bar{\phi}\left(q, q^{\prime}, s\right)$ as follows:

$$
\bar{\phi}\left(q, q^{\prime}, s\right)=\phi_{\mathrm{eq}}(q) / s+\phi_{\mathrm{eq}}(0) \bar{\tau}\left(q, q^{\prime}, s\right),
$$

where

$$
\bar{\tau}\left(q, q^{\prime}, s\right)=\left[1 / \phi_{\mathrm{eq}}(0)\right] \int_{0}^{\infty}\left[\phi\left(q, q^{\prime}, t\right)-\phi_{\mathrm{eq}}(q)\right] \exp (-s t) \mathrm{d} t .
$$

The functions $\bar{\phi}\left(q, q^{\prime}, s\right)$ represent the solvent time-scale functions [26-28]. The dependences of these functions on the two coordinates $q$ and $q^{\prime}$ for single-exponential autocorrelation functions were investigated in Ref. [25].

The wave packet motion is a convenient utility in describing the dynamics in both two- and three-surface models. They can be pictured by means of the free energy surfaces $G_{i}(q)$ for the ground $\left(S_{0}\right)$, locally excited (LE), and charge transfer

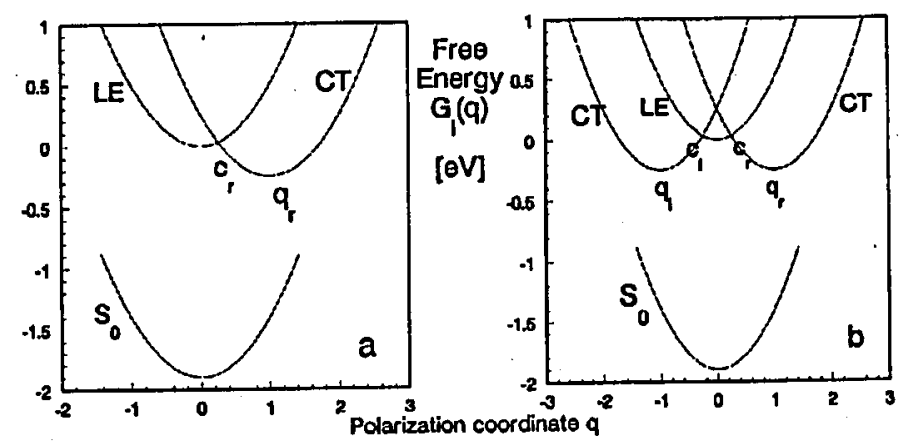

Fig. 2. The symmetrical (a) two- and (b) three-surface models of an electron transfer process in solution, where $c_{r}$ and $c_{l}$ denote the crossing points, $q_{r}$ and $q_{l}-$ the origins of parabolas. 
(CT) states as a function of polarization coordinate $q$, as described in Fig. 2. The electron transfer processes can only occur at the crossing points $c_{r}$ and $c_{l}$. Before photon excitation, the system is represented by the Boltzmann distribution in the ground state. Upon photon excitation, a population in the LE state is created. Afterwards, it will transfer from the LE state to the CT states. Here a model of nonadiabatic coupling between LE and CT states is considered using Zusman-type kinetic equations. The corresponding steady-state rate coefficients in both systems can be evaluated in the Laplace space using the adiabaticity parameter $\bar{\kappa}_{j}(0)$ or the time-scale functions $\bar{\tau}(x, y, 0)$ [21-23]

$$
\begin{aligned}
& \bar{k}_{j}(s)=2 \pi V_{r}^{2} / \hbar \frac{(j-1) \phi_{\mathrm{eq}}\left(c_{r}\right)+\phi_{\mathrm{eq}}\left(c_{r}-q_{r}\right)}{1+\bar{\kappa}_{j}(0)}, \\
& \bar{\kappa}_{j}(0)=\left(2 \pi V_{r}^{2} / \hbar\right) \phi_{\mathrm{eq}}(0)\left[\bar{\tau}\left(c_{r}, 0\right)+\bar{\tau}\left(c_{r}-q_{r}, 0\right)+(j-2) \bar{\tau}\left(c_{r}, c_{l}, 0\right)\right],
\end{aligned}
$$

where $V_{i}$ is the electronic coupling parameter at the crossing point $c_{i}$. For the case $V_{r}=V_{l}$ (symmetrical three-surface problem), $j=3$, whereas for $V_{l}=0$ and $V_{r} \neq 0$ (two-surface problem), $j=2$. A comparison of Eq. (8) for $j=3$ and 2 shows the difference between these models as a result of various adiabaticity parameters $\bar{\kappa}_{j}(0)$. Since the term $\bar{\tau}\left(c_{r}, c_{l}, 0\right)$ is negative for large separation of $c_{r}$ and $c_{l}, \bar{\kappa}_{2}(0)>\bar{\kappa}_{3}(0)$, but $\bar{\kappa}_{2}(0)<\bar{\kappa}_{3}(0)$ should hold for small separation of $c_{r}$ and $c_{l}[26]$.

Two types of the time-scale function appear in Eq. (9) for the adiabaticity parameter. The first quantity $\bar{\tau}(x, x, 0)=\bar{\tau}(x, 0)$ characterizes an escape of the wave packet from the initial position at $x$ to the equilibrium distribution. The monitoring position of the wave packet motion is also at $x$. The adiabaticity parameter $\bar{\kappa}_{j}(0)$ for the two-surface model contains two escape times $\bar{\tau}\left(c_{r}, 0\right)$ and $\bar{\tau}\left(c_{r}-q_{r}, 0\right)$ from the crossing point $c_{r}$ of two surfaces to the equilibrium distributions in each surface. The third time-scale function appearing in Eq. (9), $\bar{\tau}\left(c_{r}, c_{l}, 0\right)$ describes the dynamic interaction of two-electron transfer processes occurring at two crossing points. This situation is possible for electron transfer in dimmeric systems, where the three-surface model is applied. To reveal the influence of solvent dynamics on electron transfer, $\bar{\tau}\left(c_{r}, 0\right)$ and $\bar{\tau}\left(c_{r}, c_{l}, 0\right)$ can be investigated as the functions defining the adiabaticity parameter, for different values of the free energy $\Delta G=G_{\mathrm{LE}}-G_{\mathrm{CT}}$.

\section{Numerical calculations and discussion}

The $\Delta(t)$ functions were fitted to the results of recent MD studies $[9,12-14$, $17,20]$. For methanol a fit to two exponentials and a Gaussian function is most adequate according to the results of Fonseca and Ladanyi [20]. The long-time behaviour of $\Delta(t)$ was taken from Ref. [14]. For other cases only one exponential was used. The function for acetonitrile was taken from the work of Maroncelli [17]. In the case of water mainly the results of Maroncelli and Fleming [9] and more recent results [12-14] were taken into account. To calculate parameters for a model ET process the reorganization energies estimated from MD studies were also needed. Most of MD simulations were only concerned with modelling of solvent relaxation after instantaneous photoexcitation of various species. There were, however, several ET-MD works published [5,10,11,16,18]. Bader and Chandler [11] studied a 
realistic model of aqueous ferric-ferrous system, on the other hand, Phelps et al. [18] used a more general model of electron transfer reactions in methanol approximating reactants by a pair of Lennard-Jones spheres in contact of varying diameter and containing a univalent charge. The value for $\lambda$ of around $1.3 \mathrm{eV}$ obtained by these latter authors is most suitable for the present purpose. The values for water and acetonitrile were scaled according to the Pekar factors $\left(1 / \epsilon_{\mathrm{op}}-1 / \epsilon_{\mathrm{s}}\right)$ in the expression for $\lambda$ [29] for these solvents. The programs were written in C. The integrals in the solvent time-scale functions were evaluated using Gaussian quadrature [30]. The calculations taking into account the autocorrelation function given by Eq. (2) $\left(\Delta_{1}(t)\right)$ and the parameters from Table, were performed for methanol, acetonitrile and water. The results of these calculations are given in Figs. 3-5 and Table. They are denoted in Figs. 3-5 as "1". The results of the calculations with the fast inertial component in the solvent autocorrelation function $\Delta_{2}(t)$ and the corresponding parameters from Table are denoted as "2" in Figs. 3-5.

\subsection{Methanol}

The time-scale functions $\bar{\tau}(0,0)$ calculated using $\Delta_{1}(t)$ and $\Delta_{2}(t)$ are the same (see Table). Using the autocorrelation function $\Delta_{1}(t)$ (without the inertial component) two-exponential terms make the escape from crossing point faster $(\bar{\tau}(0,0)=196 \mathrm{fs})$ than in water $(\bar{\tau}(0,0)=284 \mathrm{fs})$ and acetonitrile $(\bar{\tau}(0,0)=707 \mathrm{fs})$. The ET rates are relatively high $\left(\bar{k}_{2}(0)=2.4 \times 10^{12} 1 / \mathrm{s}, \bar{k}_{3}(0)=3.3 \times 10^{12} 1 / \mathrm{s}\right.$; when $\lambda=\Delta G=1.3 \mathrm{eV})$. Small inertial effect $(b=0.2)$ with a high frequency solvent mode $(\omega=30.351 / \mathrm{ps})$ changes only slightly the wave packet motion (functions $\bar{\tau}\left(c_{r}, 0\right)$ and $\bar{\tau}\left(c_{r}, c_{l}, 0\right)$ ) and the rate of electron transfer $\left(\bar{k}_{2}(0)=2.7 \times\right.$ $10^{12} 1 / \mathrm{s}, \vec{k}_{3}(0)=3.8 \times 10^{12} 1 / \mathrm{s} ;$ for $\left.\Delta G=1.3 \mathrm{eV}\right)$.

\subsection{Acetonitrile}

The amplitude of variations in the time-scale functions with respect to $\Delta G$ (Fig. 3a) is smaller than for methanol, because there is only one exponential in the solvent autocorrelation function. The absolute values of $\bar{\tau}\left(c_{r}, 0\right)$ and $\bar{\tau}\left(c_{r}, c_{l}, 0\right)$ are decreased even $50 \%$ when the Gaussian function is introduced while for methanol almost no change is observed in that case. The dynamics at the crossing points is slowed down after the introduction of large inertial component $(b=0.7)$ with a low frequency solvent mode $(\omega=8.421 / \mathrm{ps})$ to $\Delta(t)$. The electron transfer rate coefficients $\bar{k}_{2}(0)$ (Fig. $4 \mathrm{a}$ ) and $\bar{k}_{3}(0)$ (Fig. 5a) for the function $\Delta_{1}(t)$ (see traces denoted " 1 ") are rather small. They can be $20-25 \%$ greater when the Gaussian component is introduced (traces " 2 ").

\subsection{Water}

The amplitude of variations in the time-scale functions with respect to $\Delta G$ (Fig. $3 \mathrm{~b}$ ) is significantly smaller than for acetonitrile, but the behaviour of the absolute values of $\bar{\tau}\left(c_{r}, 0\right)$ and $\bar{\tau}\left(c_{r}, c_{l}, 0\right)$ after the introduction of the Gaussian function is the same as for acetonitrile. They are decreased again even $50 \%$ (compare traces " 1 " and " 2 " noting that they are plotted in their own units $\bar{\tau}(0,0))$. 

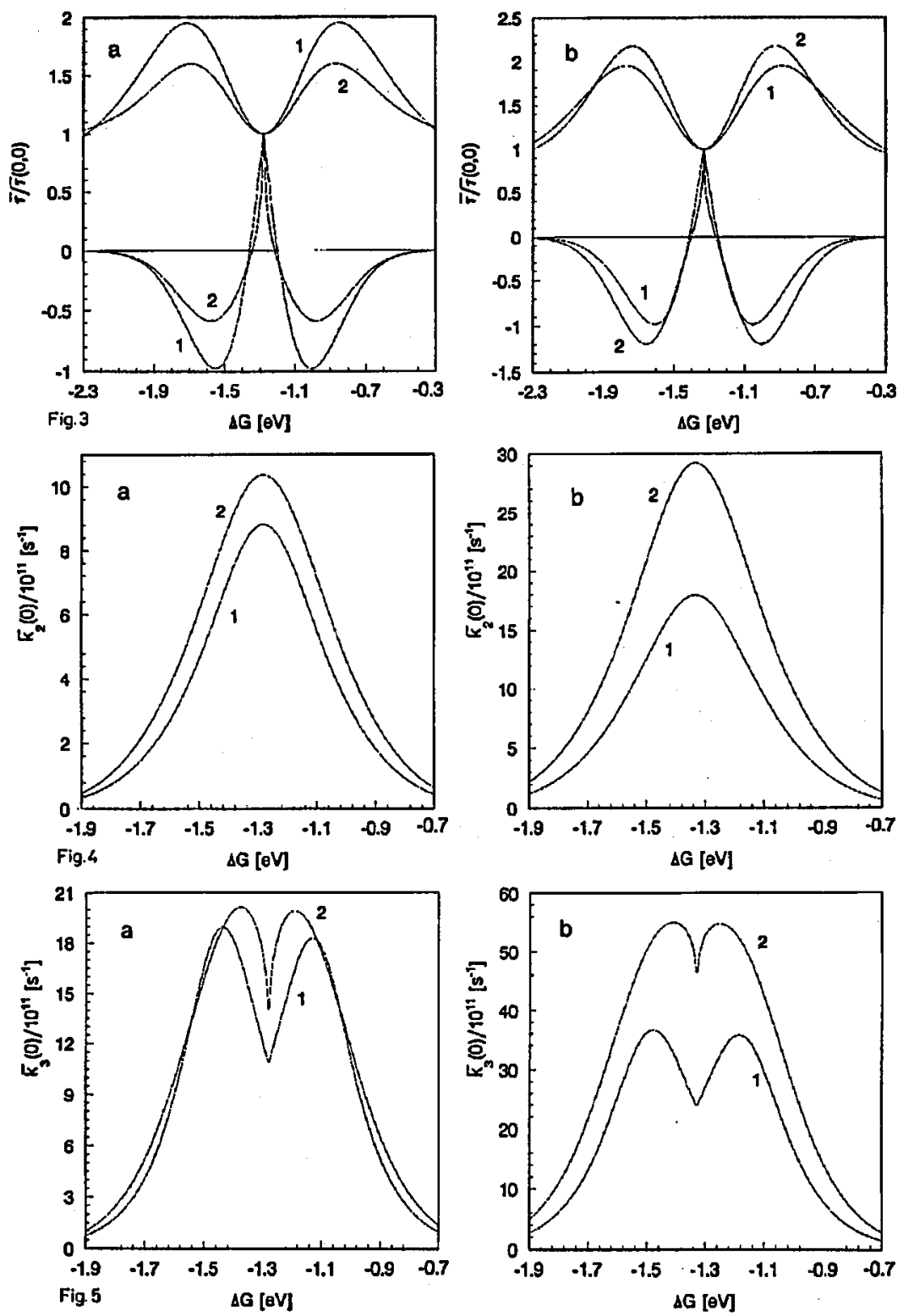

Fig. 3. The solvent time-scale functions $\bar{\tau}\left(c_{r}, 0\right) / \bar{\tau}(0,0)$ (two upper curves) and $\bar{\tau}\left(c_{r}, c_{l}, 0\right) / \bar{\tau}(0,0)$ (two lower curves) vs. free energy, $\Delta G$, of an electron transfer process for exponential solvation dynamics (1), and (2) including ultrafast inertial Gaussian component, for acetonitrile (a) and water (b).

Fig. 4. The free energy dependence of the steady-state rate coefficients in the two-surface model of an electron transfer process in solution for exponential solvation dynamics (1), and (2) including ultrafast inertial Gaussian component, for acetonitrile (a) and water (b)

Fig. 5. The free energy dependence of the steady-state rate coefficients in the three-surface model of an electron transfer process in solution. 
TABLE

The parameters used in simulations (for all cases $T=298 \mathrm{~K}$, $V=0.02 \mathrm{eV})$, and the values of $\bar{\tau}(0,0)$.

\begin{tabular}{|c|c|c|c|c|c|c|c|c|}
\hline \multirow[t]{2}{*}{ Solvent } & \multirow{2}{*}{$\begin{array}{c}\lambda \\
{[\mathrm{eV}]}\end{array}$} & \multicolumn{6}{|c|}{ Parameters in $\Delta_{1}(t)$} & \multirow{2}{*}{$\begin{array}{c}\bar{\tau}(0,0) \\
{[\mathrm{ps}]}\end{array}$} \\
\hline & & $a_{1}$ & \multicolumn{2}{|c|}{$\tau_{1}[\mathrm{ps}]$} & \multicolumn{2}{|c|}{$a_{2}$} & $\tau_{2}[\mathrm{ps}]$ & \\
\hline $\mathrm{MeOH}$ & 1.3 & 0.625 & \multicolumn{2}{|c|}{0.11} & \multicolumn{2}{|c|}{0.375} & 2.19 & 0.196 \\
\hline $\mathrm{MeCN}$ & 1.28 & 1.0 & \multicolumn{2}{|c|}{1.02} & \multicolumn{2}{|c|}{-} & - & 0.707 \\
\hline $\mathrm{H}_{2} \mathrm{O}$ & 1.33 & 1.0 & \multicolumn{2}{|c|}{0.41} & \multicolumn{2}{|l|}{ - } & - & 0.284 \\
\hline \multirow[t]{2}{*}{ Solvent } & $\lambda$ & \multicolumn{6}{|c|}{ Parameters in $\Delta_{2}(t)$} & $\overline{\bar{\tau}(0,0)}$ \\
\hline & {$[\mathrm{eV}]$} & $a_{1}^{\prime}$ & $\tau_{1}[\mathrm{ps}]$ & $a_{2}^{\prime}$ & $\tau_{2}[\mathrm{ps}]$ & $b$ & $\omega\left[\mathrm{ps}^{-1}\right]$ & {$[\mathrm{ps}]$} \\
\hline $\mathrm{MeOII}$ & 1.3 & 0.5 & 0.11 & 0.3 & 2.19 & 0.2 & 30.35 & 0.154 \\
\hline $\mathrm{MeCN}$ & 1.28 & 0.3 & 1.02 & - & - & 0.7 & 8.42 & 0.462 \\
\hline $\mathrm{H}_{2} \mathrm{O}$ & 1.33 & 0.4 & 0.41 & - & - & 0.6 & 55.23 & 0.102 \\
\hline
\end{tabular}

The behaviour of the absolute values of time-scale functions is not influenced by the change of solvent mode frequency, from low in acetonitrile to high in water $(\omega=55.231 / \mathrm{ps})$. The rate coefficients $\bar{k}_{2}(0)$ (Fig. $\left.4 \mathrm{~b}\right)$ and $\bar{k}_{3}(0)$ (Fig. 5b) are very high already for exponential solvation (traces " 1 "). They are augmented even $50 \%$ when the inertial component is included (traces "2").

The inertial effects can be observed only in the cases where the Gaussian component covers at least a half of the whole relaxation $(b=0.5 \div 0.8$, not the case of methanol). The inertial effects result in a decrease of the dynamics at the crossing points, hence in an impeding effect on the electron transfer kinetics as indicate the absolute values of the time-scale functions. Such an effect is not influenced by the increase in $\omega$. Such an increase results in faster electron transfer in both models in the case of water.

\section{Summary}

The inertial effects in ET kinetics have been modelled on the basis of recent MD simulations. The role of ultrafast inertial component of solvation dynamics in three solvents have been analyzed. These ultrafast effects are particularly important for the electron transfer processes in acetonitrile and water, where they constitute over a half of the whole solvent relaxation. They slow down the dynamics at the crossing points in both two- and three-surface models of an ET process, although it does not mean that the consideration of inertial effects results in lower ET rate coefficients. Conversely, when the Gaussian function is introduced to the solvent autocorrelation function the electron transfer rate can significantly augment, depending on the solvent vibrational frequency $\omega$.

\section{Acknowledgment}

This work was partially supported by the grant $1721 / 2 / 91$ of the Committee for Scientific Research and the Ministry of Education. Numerical calculations were run on a CONVEX C3820 computer (calculational grant KBN/UJ/038/94). The 
author would like to thank prof. Jan Najbar for introduction to the topic of this paper and stimulating discussions.

\section{References}

[1] P.F. Barbara, W. Jarzęba, Adv. Photochem. 15, 1 (1990).

[2] M. Maroncelli, G.R. Fleming, J. Chem. Phys. 86, 6221 (1987).

[3] S.J. Rosenthal, X. Xiaoliang, D. Mei, G.R. Fleming, J. Chem. Phys. 95, 4715 (1991).

[4] M.J. Weaver, G.E. McManis, W. Jarzęba, P.F. Barbara, J. Phys. Chem. 94, 1715 (1990).

[5] D.A. Zichi, G. Ciccotti, J.T. Hynes, M. Ferrario, J. Phys. Chem. 93, 6261 (1989).

[6] J.T. Hynes, E.A. Carter, G. Ciccotti, H.J. Kim, D.A. Zichi, M. Ferrario, R. Kapral, in: Perspectives in Photosynthesis, Eds. J. Jortner, B. Pullman, Kluwer Academic, Dordrecht 1990, p. 336.

[7] E.A. Carter, J.T. Hynes, J. Chem. Phys. 94, 5961 (1991).

[8] M. Maroncelli, J. Mol. Liq. 57, 1 (1993).

[9] M. Maroncelli, G.R. Fleming, J. Chem. Phys. 89, 5044 (1988).

[10] R.A. Kuharski, J.S. Bader, D. Chandler, M. Sprik, M.L. Klein, R.W. Impey, J. Chem. Phys. 89, 3248 (1988).

[11] J.S. Bader, D. Chandler, Chem. Phys. Lett. 157, 501 (1989).

[12] R.M. Levy, D.B. Kitchen, J.T. Blair, K. Krogh-Jespersen, J. Phys. Chem. 94, 4470 (1990).

[13] M. Belhadj, D.B. Kitchen, K. Krogh-Jespersen, R.M. Levy, J. Phys. Chem. 95, $1082(1991)$.

[14] K. Ando, S. Kato, J. Chem. Phys. 95, 5966 (1991).

[15] E.A. Carter, J.T. Hynes, J. Phys. Chem. 93, 2184 (1989).

[16] B.B. Smith, A. Staib, J.T. Hynes, Chem. Phys. 176, 521 (1993).

[17] M. Maroncelli, J. Chem. Phys. 94, 2084 (1991).

[18] D.K. Plielps, M.J. Weaver, B.M. Ladanyi, Chem. Phys. 176, 575 (1993).

[19] J. Zhu, R.I. Cukier, J. Chem. Phys. 98, 5679 (1993).

[20] T. Fonseca, B.M. Ladanyi, J. Phys. Chem. 95, 2116 (1991).

[21] I. Rips, J. Jortner, J. Chem. Phys. 87, 2090, 6513 (1987).

[22] J.N. Onuchic, J. Chem. Phys. 86, 3925 (1987).

[23] L.D. Zusman, Chem. Phys. 119, 51 (1988).

[24] P. Gajdek, J. Najbar, A.M. Turek, J. Photochem. Photobiol. A: Chem. 84, 113 (1994).

[25] J. Najbar, M. Boczar, A.M. Turek, Polish J. Chem. 67, 1425 (1993).

[26] J. Najbar, W. Jarzęba, Chem. Phys. Lett. 196, 504 (1992).

[27] Y.J. Yan, S. Mukamel, J. Phys. Chem. 93, 6991 (1989).

[28] T. Fonseca, J. Chem. Phys. 91, 2869 (1989).

[29] R.A. Marcus, J. Chem. Phys. 43, 679 (1965).

[30] W.H. Press, B.P. Flannery, S.A. Teukolsky, W.T. Vettering, Numerical Recipes, Cambridge Univ. Press, Cambridge 1986. 